\title{
Study on fuel consumption and emission characteristics of China VI heavy duty vehicle based on vehicle specific power
}

\author{
Dandan $\mathrm{Xu}^{1, *}$, Zhongming Gao ${ }^{1}$, Yong Guo ${ }^{1}$, Yan $\mathrm{Yan}^{1}$, Fengbin Wang ${ }^{1}$, and Fulu $\mathrm{Shi}^{2}$ \\ ${ }^{1}$ China Automotive Technology and Research Center Co., Ltd, China \\ ${ }^{2}$ Beijing Vehicle Emissions Management Center, China
}

Keywords: China VI heavy duty vehicle, vehicle specific power, fuel consumption and emission characteristics.

\begin{abstract}
This study selects a ChinaVI heavy duty vehicle for PEMS test, and Based on the measurement results of vehicle specific power (VSP) parameters, the VSP calculation formula applicable to this study is proposed, And analyzes the distribution characteristics of VSP, and at the same time according to the fuel consumption and emission data of the actual road driving process collected by the vehicle, The effect of VSP on vehicle fuel consumption and emission characteristics and the correlation between the two are studied. Results show that VSP of the vehicle are mainly concentrated in the interval $-10 \leqslant \mathrm{VSP} \leqslant 10 \mathrm{kw} / \mathrm{t}$, in which the vehicle driving time accounts for about $99.3 \%$ of the total time; the correlation coefficient between VSP and average fuel consumption is about 0.93 , there is a strong correlation; The changes of $\mathrm{CO}, \mathrm{CO}_{2}, \mathrm{NO}$ and $\mathrm{PN}$ with VSP all show as that under the same absolute value of VSP, the pollutants emission rate in the VSP $>0$ interval is higher than the VSP $<0$ interval, in which the correlation between VSP and $\mathrm{CO}, \mathrm{CO}_{2}, \mathrm{PN}$ emissions is more strong, but poorly correlated with $\mathrm{NO}_{\mathrm{x}}$ emissions.
\end{abstract}

\section{Introduction}

Because there are many types of heavy duty vehicles and a wide range of using, their actual fuel consumption and emission performance are greatly affected by various factors such as road conditions, environment, vehicle type, and vehicle weight. Simply using single variable such as speed or acceleration to analyze the vehicle's actual fuel consumption and emission performance during driving does not fully reflect the actual level of the vehicle. With the establishment and mature application of the vehicle specific power (VSP) model in light duty vehicles [4-10], the application of the VSP model that integrates the actual speed, acceleration, road and environmental parameters of the vehicle in evaluating the fuel consumption and emission performance of the motor vehicle is favored by many scholars at home and abroad. Among them, Pang Ran et al. Built the fuel consumption model of light vehicles on uphill and downhill conditions based on the VSP model and verified the model.

* Corresponding author: xudandan@catarc.ac.cn 
The results show that using specific power to calculate the fuel consumption of the vehicle has high accuracy [1]; Peng Meichun et al. Studied the impact of VSP on LPG bus emissions, and found that VSP had a greater impact on vehicle pollutant emissions [3]; Hai Wei Wang et al. Established a microscopic emission model based on VSP and effectively evaluated the vehicle's instantaneous emissions and total emissions results [2]. At present, the application research of VSP is mainly limited to light vehicles, and the research on the parameter calibration of heavy duty vehicles VSP and the establishment and application of models are still insufficient.

Based on the actual road driving conditions of ChinaVI heavy duty vehicles, this paper uses the data collected by the PEMS test to put forward the VSP calculation formula is suitable for this study, and analyzes the distribution characteristics of VSP in the test, and the impact of VSP on the vehicle's fuel consumption and emission characteristics, and the correlations between VSP and vehicle fuel consumption and emissions, in order to establish suitable for heavy duty vehicle VSP model provides theoretical basis.

\section{Vehicle Specific Power (VSP)}

Motor vehicle specific power $(\mathrm{kW} / \mathrm{t})$ is the power that characterizes the output of the engine to overcome the rolling resistance, air resistance, ramp resistance and acceleration resistance of the vehicle during driving. Based on the speed, it comprehensively considers the influence of various factors such as wind resistance, road gradient, acceleration, environmental conditions and other factors on the vehicle's fuel consumption and emissions during driving. Using VSP to analyze the vehicle's fuel consumption and emissions characteristics can not only effectively avoid the impact of vehicle load state change on fuel consumption and emission results, but also can more truly reflect the actual road driving characteristics of the vehicle.VSP is a vector variable with direction. When VSP $>0$, it indicates that the vehicle is in the acceleration driving condition, otherwise, the vehicle is in the deceleration condition. When VSP $=0$, it indicates that the vehicle is in the idle mode [11]. According to the definition of VSP, the calculation formula is characterized as follows:

$$
\mathrm{VSP}=\mathrm{v} \cdot[\mathrm{g} \cdot \mathrm{f}+\mathrm{g} \cdot \sin \alpha+(1+\varepsilon) \cdot \mathrm{a}]+0.5 \rho \frac{\mathrm{\tau} \cdot \mathrm{A}}{\mathrm{m}}\left(\mathrm{v}+\mathrm{v}_{\mathrm{m}}\right)^{2} \cdot \mathrm{v}
$$

In the formula: $\mathrm{v}$ is the vehicle's driving speed, $\mathrm{km} / \mathrm{h}$; $\mathrm{g}$ is the acceleration of gravity, with a value of $9.8 \mathrm{~m} / \mathrm{s}^{2}$; $\mathrm{f}$ is the rolling resistance coefficient; $a$ is the road slope, with a value of $0^{\circ} ; \varepsilon$ is the quality factor, with a value of 0.1 ; a is the vehicle's driving acceleration, $\mathrm{m} / \mathrm{s}^{2} ; \rho$ is the density in the experimental environment, $\mathrm{g} / \mathrm{m}^{3} ; \quad \tau$ is the air resistance coefficient, $\mathrm{A}$ is the windward area of the vehicle, $\mathrm{m}^{2} ; \mathrm{v}_{\mathrm{m}}$ is the wind speed in the experimental environment.

\section{Experimental design and scheme}

\subsection{Test design}

The test selects a section of straight road with good road conditions at an ambient temperature of $27^{\circ} \mathrm{C}$, an average atmospheric pressure of $101 \mathrm{kpa}$, and a wind speed of $2 \mathrm{~m}$ / s, according to GB 17691-2018 "Limits and Measurement Methods for Pollutant Emissions of Heavy Diesel Vehicles (China VI stage)"Using the portable emission measurement system (PEMS) made by Horiba to carry out actual road emission test on a 
fully loaded $\mathrm{N}_{3}$ city vehicle. In the test, the driving conditions of the vehicle are $20 \%$ of the urban condition, $25 \%$ of the rural condition and $55 \%$ of the motorway condition. Figure 1 shows the driving conditions of vehicle during the test.

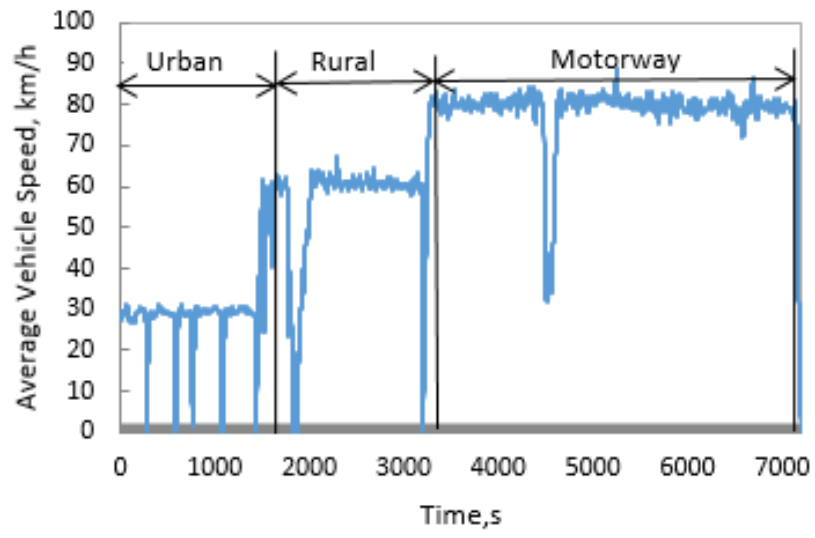

Fig. 1. Driving condition.

At the same time, the test is based on GB 27840-2011 "Heavy Commercial Vehicle Fuel Consumption Measurement Method" Appendix C [12], using a barometer, density meter and other measurement equipments to conduct a static measurement test of the VSP parameter of the vehicle before the PEMS test begins, According to the standard and the calculation method of running resistance stipulated in the standard, the parameters required in formula (1) are calculated by using the measurement results of static test, as shown in Table 2. The basic information of the vehicle and engine selected in the experiment is shown in Table 1.

Table 1. Basic information of vehicle.

\begin{tabular}{cc}
\hline Vehicle parameters & Basic Information \\
\hline Gross vehicle weight $(\mathrm{kg})$ & 31000 \\
Tyre type & Radial Tire \\
Vehicle dimensions $(\mathrm{mm})$ & $10150 \times 2550 \times 3980$ \\
Vehicle minimum ground clearance $(\mathrm{mm})$ & 320 \\
Emission level & $\mathrm{ChinaVI}$ \\
Engine power / Displacement $(\mathrm{kW} / \mathrm{L})$ & $257 / 7.8$ \\
After-treatment type & $\mathrm{SCR}+\mathrm{DOC}+\mathrm{DPF}+\mathrm{ASC}$ \\
\hline
\end{tabular}

\subsection{Test Scheme}

Using the VSP parameters value in Table 2, the formula (1) is simplified to get the formula (2) of the heavy duty vehicle VSP is suitable for this study according to the vehicle driving data collected by the experiment, the instantaneous VSP value can be calculated by using formula (2), and analyze the distribution characteristics of the VSP during the driving of the vehicle by using data statistics methods, and at the same time, based on the driving data and emission data collected by the experiment, the carbon balance method is used to calculate the instantaneous fuel consumption of the vehicle, and analyze the influence of VSP on the vehicle fuel consumption and emissions, as well as the correlation between VSP and fuel consumption and emissions.

$$
\operatorname{VSP}=\mathrm{v} \cdot(1.1 \mathrm{a}+0.049)+0.00014 \cdot \mathrm{v}^{3}
$$

Table 2 VSP parameter values 


\begin{tabular}{cc}
\hline VSP Parameters & Values \\
\hline Rolling resistance coefficient & 0.005 \\
Air density $\left(\mathrm{g} / \mathrm{m}^{3}\right)$ & 1.156 \\
Frontal area $\left(\mathrm{m}^{2}\right)$ & 9.33 \\
Air resistance coefficient & 0.8 \\
Environmental wind speed $(\mathrm{m} / \mathrm{s})$ & 1.6 \\
\hline
\end{tabular}

\section{Analysis of test results}

\subsection{VSP distribution characteristics}

Through the VSP of the vehicle by the second of data statistics, and it is found that the operating points of the vehicle during the driving process are mainly distributed in VSP $\in$ $[-10,10]$. The VSP is divided into equal intervals at intervals of $2 \mathrm{kw} / \mathrm{t}$, and the distribution of VSP in the actual driving process of the vehicle is analyzed, as shown in Figure 2. Since the idling time of the vehicle in the experiment is less than $1 \%$, the $\mathrm{VSP}=0$ interval is not divided separately in this paper.

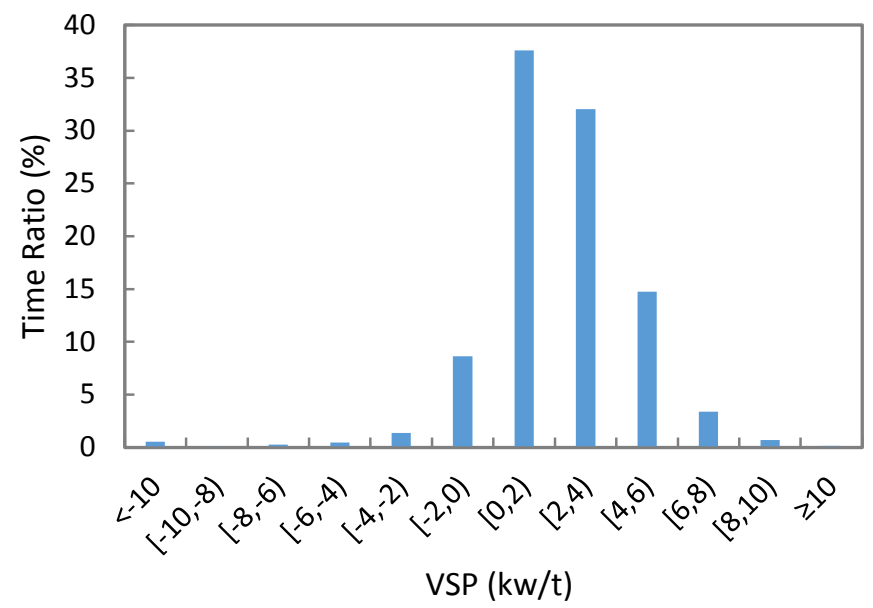

Fig. 2. VSP distribution characteristics.

Figure 2 shows that in the VSP $\in[-10,10]$ interval, vehicle driving time accounts for $99.3 \%$ of the total time, of which the VSP in the $[0,2)$ interval accounts for the highest proportion, $37.6 \%$. The vehicle runs more in the VSP $>0$ interval, which accounts for about $88.7 \%$ of the total time. As the VSP increases, the time ratio gradually decreases, indicating that the vehicle has more running time under low acceleration conditions, less running time under deceleration conditions. The analysis results are consistent with the driving characteristics of the vehicle in this experiment (as shown in Figure 1), that is, the running time in urban conditions is short, the running time in high-speed conditions is long, Therefore, there are relatively few start-stop conditions and idle speed conditions during the vehicle driving. 


\subsection{Analysis of the influence of VSP on vehicle fuel consumption}

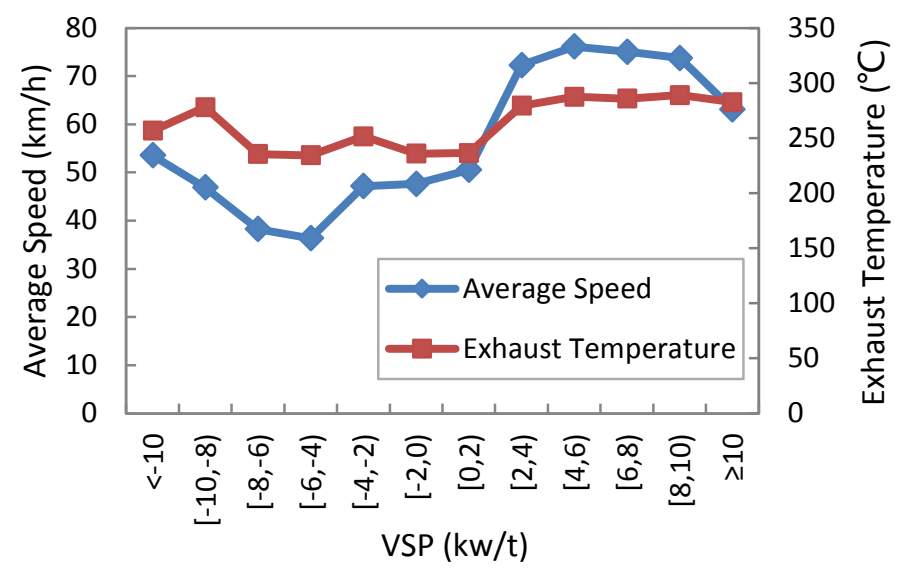

Fig. 3. VSP and average vehicle speed, average exhaust temperature.

Figure 3 shows how the average vehicle speed and average exhaust temperature change with VSP. When VSP $\geqslant 2 \mathrm{kw} / \mathrm{t}$, the average speed and average exhaust temperature of the vehicle are both high, it indicates that the vehicle in this interval drives more at high speed. It can be seen from Figure 1 that the ratio of driving time in this interval is about $51.1 \%$, VSP is in the interval of $-8 \sim 0 \mathrm{kw} / \mathrm{t}$, the average speed is less than $50 \mathrm{~km} / \mathrm{h}$, the average exhaust temperature is low and the change is stable, indicating that the vehicle in this interval is driving more at low speeds, and from Figure 1 we can see that the vehicle driving time in this interval is about $10.7 \%$.

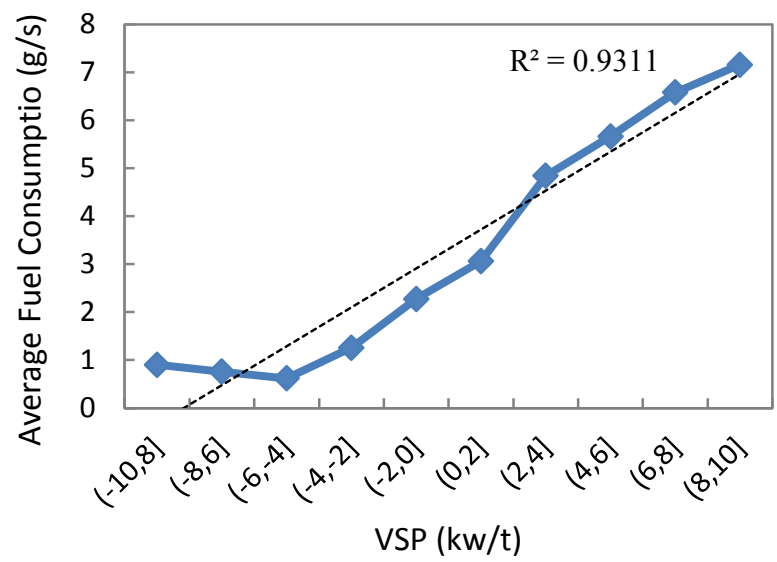

Fig. 4. VSP and average fuel consumption.

The experiment uses the carbon balance method to analyze and calculate the instantaneous fuel consumption of the vehicle during driving, and divides the main distribution intervals of driving conditions from -10 to $10 \mathrm{kw} / \mathrm{t}$ into 10 partitions at intervals of $2 \mathrm{kw} / \mathrm{t}$. Calculate the average fuel consumption of 10 intervals respectively, and analyze the impact and the correlation between VSP and vehicle average fuel consumption. Figure 4 shows the change of the average fuel consumption with VSP and the correlation between VSP and average fuel consumption. It can be seen from the figure 4 that the average fuel consumption of the vehicle in the acceleration section of VSP $>0$ is 
higher than that in the deceleration interval of VSP $<0$. When the vehicle is in the interval of $-10 \sim-4 \mathrm{kw} / \mathrm{t}$, the average fuel consumption of the vehicle decreases slowly with the decrease of the absolute value of VSP. and when the VSP is in the interval of $-4 \sim 0 \mathrm{kw} / \mathrm{t}$, the average fuel consumption of the vehicle increases continuously with the decrease of the absolute value of VSP. When the VSP $>0$ interval, the average fuel consumption continues to increase with the increase of VSP, so in the $-6 \sim-4 \mathrm{kw} / \mathrm{t}$ interval, the average fuel consumption of the vehicle is the lowest, about $0.6 \mathrm{~g} / \mathrm{s}$. At the same time, Figure 4 shows that the correlation between VSP and fuel consumption during vehicle driving is high, and the correlation coefficient is about 0.93 .

\subsection{Analysis of the impact of VSP on vehicle emissions}

Similar to fuel consumption analysis, VSP $\in[-10,10]$ is used as the emission analysis interval, and the interval is divided at $2 \mathrm{kw} / \mathrm{t}$ intervals to analyze the emission characteristics of the four pollutants $\mathrm{CO}, \mathrm{CO}_{2}, \mathrm{NO}_{\mathrm{X}}, \mathrm{PN}$ with VSP during the driving process of the vehicle.

\subsection{1 $\mathrm{CO} A N D \mathrm{CO}_{2}$ emission characteristics}

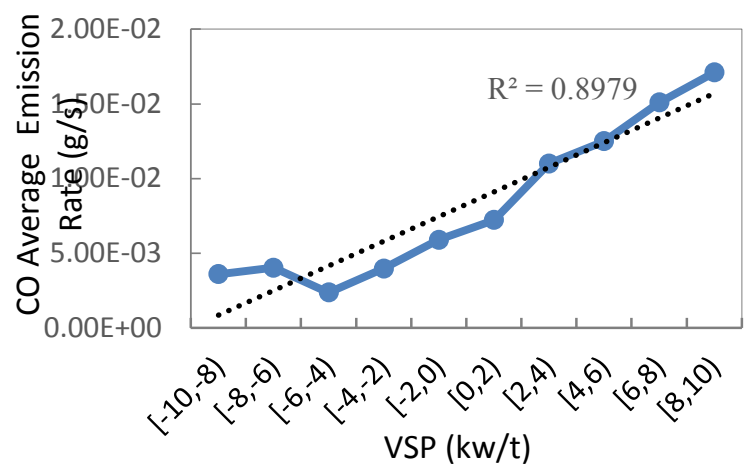

Fig. 5. VSP and CO emission.

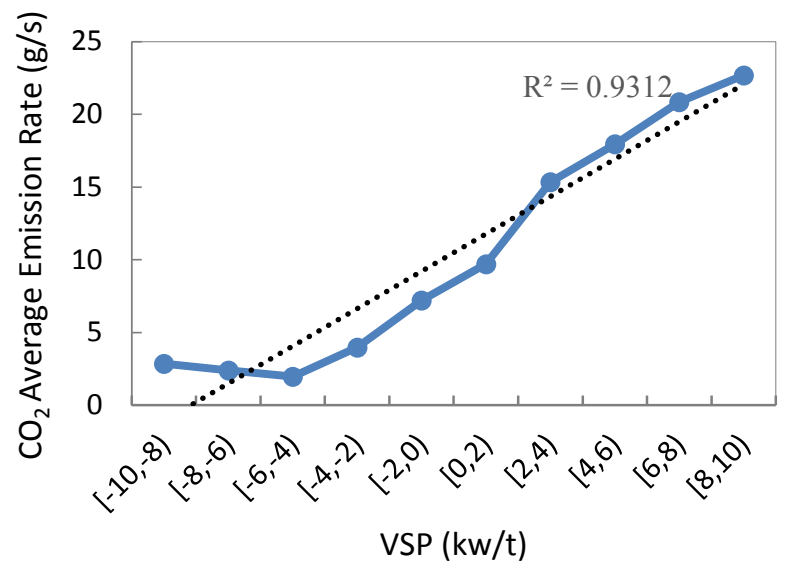

Fig. 6. VSP and $\mathrm{CO}_{2}$ emission. 
Figure 5 and Figure 6 show the change rule of the average $\mathrm{CO}$ and $\mathrm{CO}_{2}$ emission rates with VSP. The change rule of $\mathrm{CO}_{2}$ with VSP is similar to fuel consumption. When VSP $<0$, with the decrease absolute value of VSP, the $\mathrm{CO}_{2}$ emission decreases slowly and then gradually increases, and the emission rate is the lowest in the interval of $-6 \sim-4 \mathrm{kw} / \mathrm{t}$, about $0.002 \mathrm{~g} / \mathrm{s}$. In the interval of $-10 \sim-4 \mathrm{kw} / \mathrm{t}$, the average CO emission rate increases firstly and then decreases as the absolute value of VSP decreases. In the interval of $-4 \sim 0 \mathrm{~kW} / \mathrm{t}$, the $\mathrm{CO}$ emission rate gradually increases as the absolute value of VSP decreases. However, in the interval of VSP $>0$, the $\mathrm{CO}$ emission rate gradually increases with the increase of VSP. At the same time, the changes of $\mathrm{CO}$ and $\mathrm{CO}_{2}$ with VSP both show that the vehicle's emission rate in acceleration mode is higher than that in deceleration mode. Correlation analysis between VSP and $\mathrm{CO}$ and $\mathrm{CO}_{2}$ emissions shows that VSP is highly correlated with both emissions. Among them, the correlation between VSP and $\mathrm{CO}_{2}$ emission is higher, with a correlation coefficient of about 0.93 .

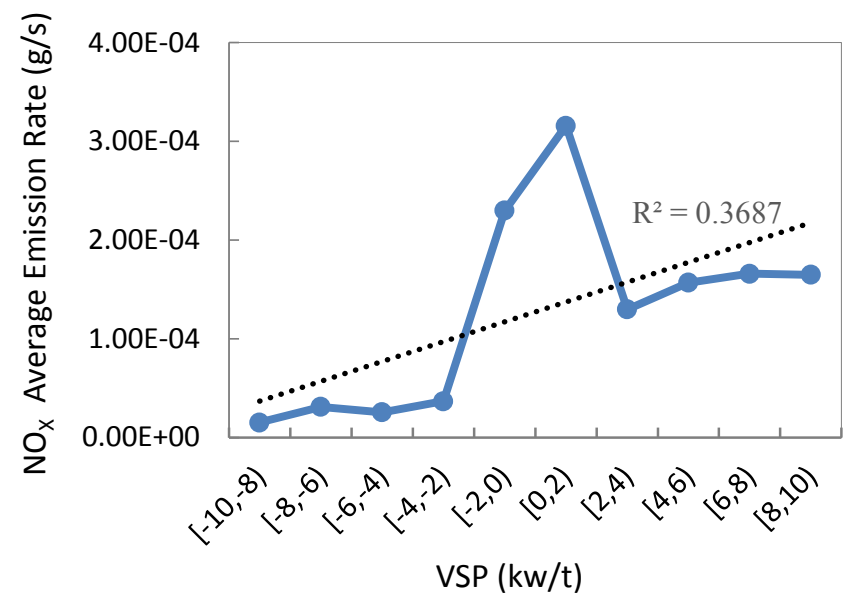

Fig. 7. VSP and $\mathrm{NO}_{\mathrm{x}}$ emission.

During vehicle driving, the $\mathrm{NO}_{\mathrm{x}}$ emission rate is low and the $\mathrm{NO}_{\mathrm{x}}$ emission amount is low. The VSP is in the interval of $-2 \sim 2 \mathrm{kw} / \mathrm{t}$, and the average $\mathrm{NO}_{\mathrm{X}}$ emission rate is relatively high. Figure 3 analyzes the change rule of the average $\mathrm{NO}_{\mathrm{X}}$ emission rate with VSP. It can be seen that the driving time of the vehicle in the interval of $-2 \sim 2 \mathrm{kw} / \mathrm{t}$ is about $46.2 \%$, and the average speed is less than $50 \mathrm{~km} / \mathrm{h}$. The average exhaust temperature of the vehicle is lower than the average exhaust temperature of the entire driving process. Therefore, when the vehicle drives in this interval, it mainly runs at low speed, resulting in high NOx emission and high average emission rate. In the case of the same absolute value of VSP, the average $\mathrm{NO}_{\mathrm{x}}$ emission rate in the interval of VSP $>0$ is higher than that in the interval of VSP $<0$. The change rule of $\mathrm{NO}_{\mathrm{X}}$ average emission rate with VSP shows that the correlation between the two is poor, and the correlation coefficient is about 0.37 . 


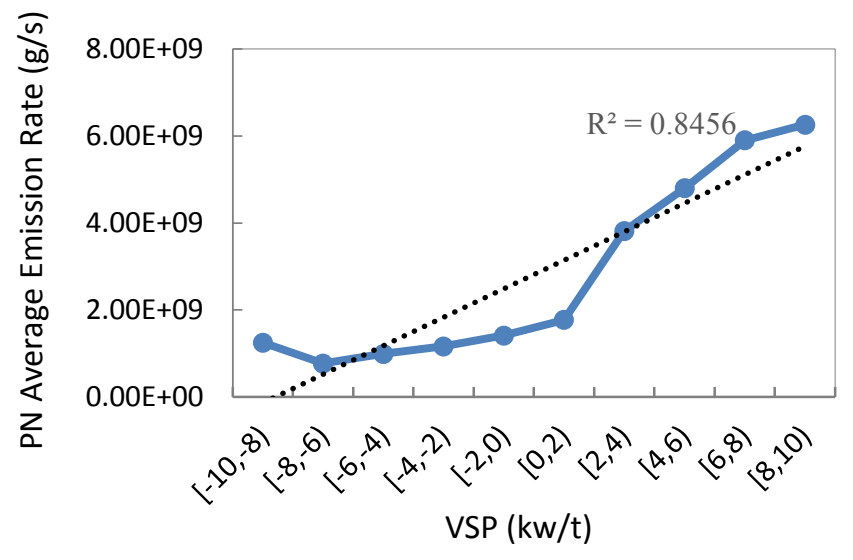

Fig. 8. VSP and PN emission.

Figure 8 shows that when VSP $<0$, as the absolute value of VSP increases, the average PN emission rate first decreases slightly and then slowly increases, and the average PN emission rate changes smoothly, while in the interval of VSP $>0$, as the absolute value of VSP increases, The average emission rate of PN increased sharply, and the average emission rate of PN changed significantly. The average PN emission rate of VSP in the $2 \sim$ $4 \mathrm{kw} / \mathrm{t}$ interval is about 2 times the average PN emission rate in the $0 \sim 2 \mathrm{kw} / \mathrm{t}$ interval, and the time ratio of VSP in the two intervals is only about $5 \%$ different. It can be seen that the VSP change from $0 \sim 2 \mathrm{kw} / \mathrm{t}$ to $2 \sim 4 \mathrm{kw} / \mathrm{t}$, and the average driving speed of the vehicle suddenly increased from $50.6 \mathrm{~km} / \mathrm{h}$ to $72.3 \mathrm{~km} / \mathrm{h}$, and he average exhaust temperature also increased from $236^{\circ} \mathrm{C}$ to $279^{\circ} \mathrm{C}$, resulting in a sudden increase in the average PN emission rate. According to the correlation analysis of VSP and the average emission rate of PN, there is a strong correlation between the two, and the correlation coefficient is about 0.85 .

\section{Conclusion}

Using a China VI heavy duty diesel vehicle as the research object, the PEMS test of the actual road driving of the vehicle was carried out, and the VSP calculation formula suitable for this study was proposed, and draw corresponding conclusions:

(1) The vehicle driving conditions are mainly concentrated in the interval $-10 \leqslant \mathrm{VSP} \leqslant$ $10 \mathrm{kw} / \mathrm{t}$, and its time ratio is about $99.3 \%$ of the total driving time. Among them, the VSP> 0 interval, that is, the running time of the vehicle under accelerated operating conditions accounts for the driving time. $88.7 \%$.

(2) When VSP $\geqslant 2 \mathrm{kw} / \mathrm{t}$, the average driving speed and average exhaust temperature are higher. VSP $\in(-6,0)$ vehicle fuel consumption gradually increases as the absolute value of VSP decreases. In the interval VSP $>0$, vehicle fuel consumption gradually increases as VSP increases, and there is a strong correlation between the two. The coefficient is about 0.93 .

(3) The change rule of the average emission rate of $\mathrm{CO}, \mathrm{CO}_{2}, \mathrm{NO}_{\mathrm{X}}$ and $\mathrm{PN}$ with VSP shows that under the same absolute value of VSP, the average emission rate of pollutants in the interval of VSP $>0$ is higher than the average emission rate in the interval of VSP $<0$.

(4) The changes of $\mathrm{CO}$ and $\mathrm{CO}_{2}$ with VSP are similar, showing that the average emission rate of the vehicle in acceleration mode is higher than the average emission rate of deceleration mode, and there is a strong correlation with VSP. The correlation coefficients are about 0.90 and 0.93 , respectively; In the range of $2 \sim 2 \mathrm{kw} / \mathrm{t}$, the vehicle average speed 
is less than $50 \mathrm{~km} / \mathrm{h}$, the average $\mathrm{NO}_{\mathrm{X}}$ emission rate is high, and the correlation between $\mathrm{NO}_{\mathrm{X}}$ emission and VSP is poor; VSP changes from $0 \sim 2 \mathrm{kw} / \mathrm{t}$ to $2 \sim 4 \mathrm{kw} / \mathrm{t}$ The average vehicle speed and emissions increase sharply, and the PN emission rate increases by about two times. The correlation between PN emission and VSP is strong, and the correlation coefficient is about 0.85 .

\section{Reference}

1. Pang Ran, Jian Xiaochun, Meng Xiong, Jin Duo. Comprehensive power consumption model of light vehicle specific power based on typical mountain cities [J] .Science Technology and Engineering, 2018,18 (06): 156-161.

2. Hai Wei Wang, Hui Ying Wen, Feng You Gui Feng Yang. Research on VSP Microcosmic Emission Model and Numerical Simulation for Motor Vehicle [J]. Key Engineering Materials, 2014, 2633.

3. Peng Meichun, Wu Xiaowei, Jiang Xiaoyan. Research on Emission Characteristics of LPG Buses Based on Specific Power [J] .Automotive Engineering, 2014, 36 (03): $269-272+263$

4. Hao Yanzhao, Yu Lei, Song Guohua, Xu Yaofang, Wang Hongtu. Study on the driving and emission characteristics of Beijing diesel buses based on specific power parameters [J]. Automotive Engineering, 2010, 32 (02): 103-109.

5. Modelling of the fuel consumption for passenger cars regarding driving characteristics [J] . Haikun Wang, Lixin Fu, Yu Zhou,He Li. Transportation Research Part D. 2008 (7)

6. Distribution Characteristics of Vehicle-Specific Power on Urban Restricted-Access Roadways[J] . Guohua Song, Lei Yu, Zhao Tu. Journal of Transportation Engineering. $2012(2)$

7. Estimation of Fuel Efficiency of Road Traffic by Characterization of Vehicle-Specific Power and Speed Based on Floating Car Data [J]. Guohua Song, Lei Yu. Transportation Research Record. 2009 (1)

8. Modeling of vehicle specific power and instantaneous speed distribution for expressways in Beijing. Xu Y, Yu L, Song G H. Proceeding of the 90th Transportation Research Board Annual Meeting . 2011

9. Delay Correction Model for Estimating Bus Emissions at Intersections Based on VSP Distributions. Zhou X, Song G, Yu L. Transportation Research Board 93rd Annual Meeting . 2014

10. Randall Guensler, Haobing Liu Yanzhi (Ann) Xu Alper Akanser, Daejin Kim Michael P. Hunter, Michael O. Rodgers. Energy Consumption and Emissions Modeling of Individual Vehicles [J]. Transportation Research Record, 2017, 2627(1).

11. Feng Hongjing, Liu Rongchang, Shi Huiwei. Parameter calibration and fuel consumption / emission calculation of heavy vehicle specific power in MOVES model [J] .Light Vehicle Technology, 2016 (Z1): 3-6 + 18.

12. GB / T 27840-2011. Measurement method of fuel consumption of heavy commercial vehicles [S]. 2011 